\title{
Interesse dos Estudantes pela Medicina de Família: Estado da Questão e Agenda de Pesquisa
}

\author{
PALAVRAS-CHAVE \\ - Medicina de Família e \\ Comunidade. \\ - Internato e residência. \\ - Escolha da profissão.
}

\section{Undergraduate Medical Students' Interest in Family Medicine: Current Issues and a Research Agenda}

Pedro Gomes Cavalcante Neto Geison Vasconcelos Liral Alcides Silva de Miranda

\section{RESUMO}

Com a criação e a expansão do Programa Saúde da Família no Brasil, a Medicina de Família e Comunidade (MFC), como especialidade médica, ganhou destaque por ser a mais adequada a esse trabalho. Recentes parcerias entre os ministérios da Saúde e da Educação têm procurado regular a formação de recursos humanos em saúde para atender às demandas de consolidação do Sistema Único de Saúde (SUS) no âmbito da graduação e da pós-graduação. Houve investimento no aumento no número de vagas de residência em MFC, mas parece haver uma incongruência entre o que os futuros médicos almejam em suas carreiras e as necessidades do SUS, o que é demonstrado pelo número de vagas ociosas nesses programas. Com o objetivo de elencar hipóteses explicativas do desinteresse por essa especialidade, fizemos uma revisão de trabalhos que enfocaram essa temática. Encontramos que pouco prestígio, baixos salários, pouca vivência em atenção primária durante a graduação e elevada dívida com a universidade foram os fatores mais recorrentes. Concluímos que é necessário investigar essas hipóteses em nossa realidade, motivo pelo qual propomos uma agenda de pesquisa nessa direção.

\section{KEYWORDS}

- Family Practice.

- Internship and residency.

- Career choice.

Recebido em: 22/11/2007

Reencaminhado em: 14/05/2008

Aprovado em: 15/07/2008 


\section{INTRODUÇÃO}

A Medicina de Família e Comunidade (MFC) é uma especialidade médica reconhecida pela Associação Médica Brasileira (AMB) e pelo Conselho Federal de Medicina (CFM). Ela é eminentemente clínica e desenvolve, de forma integrada e integradora, práticas de promoção, proteção e recuperação da saúde dirigidas a pessoas, famílias e comunidades ${ }^{1}$.

São características/atributos da MFC: primeiro ponto de contato médico com o sistema de saúde; coordenação da prestação de cuidados; abordagem centrada na pessoa, orientada para o indivíduo, a família e a comunidade; relação médicopaciente ao longo do tempo; processo de tomada de decisão determinado pela prevalência e incidência de doença na comunidade; gestão simultânea dos problemas, tanto agudos como crônicos, dos pacientes individuais; promoção da saúde e do bem-estar mediante intervenções apropriadas e efetivas; responsabilidade específica pela saúde da comunidade; levar em conta os problemas de saúde em todas as suas dimensões - física, psicológica, social, cultural e existencial; e gestão da doença que se apresenta de forma indiferenciada, numa fase precoce de sua história natural ${ }^{2}$.

No seu início, a MFC (em alguns países, apenas Medicina de Família) enfrentou dificuldades para se estabelecer como especialidade. Na Inglaterra, Canadá e Estados Unidos, ela já desfruta de algum reconhecimento no campo médico, mas pouca valorização da carreira, insuficiente qualificação profissional, baixa remuneração, pouco prestígio social e deficiência de programas de pós-graduação stricto sensu foram obstáculos que precisaram ser superados ${ }^{3,4,5}$.

No Brasil, os primeiros programas de residência afins foram em Medicina Geral e Comunitária (MGC), organizados a partir de 1976 no Rio Grande do Sul e reconhecidos pela Comissão Nacional de Residência Médica (CNRM) em 1981. Nesse ano, foi fundada a Sociedade Brasileira de Medicina Geral e Comunitária (SBMGC). Cinco anos mais tarde, a MGC foi reconhecida pelo CFM como especialidade médica. Entretanto, sua fragilidade é percebida pelas diversas ativações e desativações de sua sociedade de especialidade. A SBMGC, em 2001, passa a chamar-se Sociedade Brasileira de Medicina de Família e Comunidade (SBMFC), sendo a MFC aceita como especialidade pelo CFM, sob esta denominação, em 2003 6,7,8.

Nos últimos cinco anos, a SBMFC cresceu e consolidou-se, tendo apresentado aumento significativo do número de sócios. Também conquistou espaço junto a entidades como o CFM, a CNRM, a AMB, a Federação Nacional dos Médicos (Fenam), a Organização Pan-Americana da Saúde (Opas), a World Organization of Family Doctors (Wonca), a Confederação IberoAmericana de Medicina Familiar e o Ministério da Saúde 6 .
Contudo, foi com a criação e a expansão do Programa Saúde da Família (PSF) que a MFC ganhou destaque, sendo incorporada às políticas públicas de saúde no País. O PSF foi inicialmente formulado como programa vertical para as regiões Norte e Nordeste, com o objetivo de barrar a epidemia de cólera no início dos anos 1990. Desta feita, em janeiro de 1994 foram criadas as primeiras equipes de PSF, incorporando e ampliando a atuação dos agentes comunitários de saúde? Desde então, e principalmente a partir de 1998, o PSF deixou de ser um programa para populações excluídas do consumo de serviços de saúde, para ser considerado uma estratégia de mudança do modelo de atenção à saúde no Sistema Único de Saúde (SUS) ${ }^{10,11}$. Nesse sentido, ela tem demandado políticas educacionais no âmbito da graduação e da pós-graduação para a formação de recursos humanos com perfil adequado à consecução da estratégia.

No âmbito da graduação, houve uma grande discussão em torno do currículo das escolas médicas, que não estava em harmonia com a mudança assistencial defendida. No início deste século, as Faculdades de Medicina já haviam realizado reformas curriculares, aprofundando o enfoque em Atenção Básica à Saúde (ABS).

A trajetória brasileira de consolidação da MFC como especialidade médica, tal como apresentada, partindo da necessidade de reorganização do sistema de saúde para o tensionamento do aparelho formador, questionando-lhe a adequação dos currículos profissionais, também tem sido observada em outros países, como a Espanha ${ }^{12,13}$.

A educação médica no mundo ocidental tem passado, recentemente, por uma transição matricial que vai do paradigma flexneriano para o paradigma das competências ${ }^{14}$. Incorporando essa transição de matriz paradigmática e após um longo movimento de mudanças no campo da educação médica, diversas propostas de intervenção nos processos de formação de recursos humanos em saúde têm sido reconhecidas como indutoras de uma nova missão social das escolas médicas nos cursos de graduação e de pós-graduação ${ }^{15-22}$. A Resolução CNE/CES no 04/2001²3, que institui as Diretrizes Curriculares Nacionais para os Cursos de Graduação em Medicina (DCN-Medicina), estabeleceu o novo perfil do egresso dos cursos de graduação em Medicina, com competências em conhecimentos, habilidades e atitudes que procuram estabelecer parâmetros para a formação médica em consonância com as necessidades de saúde da população brasileira.

Nesse desiderato, recentes parcerias entre os ministérios da Saúde e da Educação têm procurado regular a formação de recursos humanos em saúde para atender às demandas de consolidação do SUS ${ }^{24,25}$, entre as quais está situada a formação 
de recursos humanos para comporem a Estratégia Saúde da Família, que, no âmbito da graduação, enseja uma educação médica baseada na comunidade ${ }^{26}$.

Com efeito, é evidente que a formação pautada no hospital não mais atende aos requisitos do novo modo de organização e gestão das práticas de saúde, fazendo com que os profissionais já envolvidos no PSF busquem aprimorar seus conhecimentos na área em programas de educação continuada e de pós-graduação. Contudo, o cenário é estarrecedor, visto haver "pouca qualificação dos profissionais [no PSF] [...] cerca de 70,0\% dos médicos e enfermeiros que atuam na saúde da família não possuem nenhuma formação de pós-graduação"27.

Em 16 de julho de 2007, de acordo com o Cadastro Nacional de Estabelecimentos de Saúde (CNES), 31.188 médicos atuavam no PSF. Entretanto, conforme a SBMFC, havia 604 médicos titulados, sugerindo pouco preparo para atuação neste nível de atenção.

Com o objetivo de reverter esse quadro, em 2005 houve grande incentivo à ampliação de residências de $\mathrm{MFC}^{28}$. Em 2006, no Ceará, foram abertas 80 novas vagas, totalizando 98 vagas. A Tabela 1 compara as vagas em MFC com as de outras especialidades básicas.

\section{TABELA 1}

Número de vagas para R1 por especialidade. Ceará, 2007

\begin{tabular}{lc}
\hline Especialidade & Vagas \\
\hline Clínica Médica & 26 \\
Pediatria & 28 \\
Cirurgia Geral & 20 \\
Ginecologia-Obstetrícia & 16 \\
Medicina de Família e Comunidade & 98 \\
\hline
\end{tabular}

Fonte: Sistema CNRM disponível em: http:/ / mecsrv04.mec.gov.br/sesu/ SIST_CNRM/APPS/cons_res_inst.asp).

Acesso em 17 jul. 2007

Apesar da escassez do número de vagas de residência em geral, pois a quantidade de egressos é elevada, há ociosidade nas residências de MFC. Em 2006, em Sobral, município do interior do Ceará, foram ocupadas quatro das 12 vagas. A segunda turma conta apenas com um residente. Em Fortaleza a situação é semelhante. Isso nos leva a pensar, de antemão, que os médicos recém-formados e, por conseguinte, os estudantes de Medicina não se sentem atraídos pela MFC, como sugerido, por exemplo, em um estudo realizado no Ceará ${ }^{29}$, Estado tido como pioneiro no PSF. Ademais, é sabido que há escolha precoce da especialidade durante o curso de Medicina, e raramente esta é $\mathrm{MFC}^{30}$.

Assim, parece haver uma incongruência entre o que os futuros médicos almejam em suas carreiras e as necessidades do Sistema de Saúde, às quais as reformas curriculares têm tentado dar resposta.

Algumas das soluções encontradas internacionalmente para o fortalecimento da MFC estão sendo ensaiadas no Brasil, entre as quais: a acreditação de programas de formação e de reconhecimento da especialidade (residências, pós-graduação stricto sensu, educação continuada, certificação do médico de família); melhoria das condições de trabalho e estímulo à pesquisa na atenção básica; melhoria salarial; e presença da Medicina de Família nas universidades ${ }^{3,4,5}$.

Contudo, são necessários estudos que visem descrever o problema em sua complexidade, para, identificados os fatores que influenciam o interesse dos estudantes de Medicina em optar pela MFC como especialidade, estabelecer estratégias no âmbito das políticas de saúde e de educação médica eficazes e efetivas para atacá-lo.

Mundo afora, há uma redução do ingresso em residências de Medicina de Família. Nos Estados Unidos, de 1997 a 2005, 480 vagas foram fechadas, o que representa uma queda de $14,7 \%$. Mesmo assim, a ociosidade subiu de $10,9 \%$ para $17,6 \%$. Mais: a presença de residentes formados no próprio país caiu de $71,7 \%$ para $40,7 \%$, o que demonstra desinteresse, uma vez que para os graduados no exterior são oferecidas apenas as vagas remanescentes ${ }^{31}$. Situação similar é vista em outros países, como Austrália ${ }^{32}$, Grécia ${ }^{33}$, Portugal ${ }^{34}$ e Canadá ${ }^{35}$. A literatura internacional é rica em estudos sobre fatores que motivam a escolha da residência.

Nosso objetivo é, pois, identificar, a partir da literatura internacional, o estado da questão concernente às principais variáveis associadas ao grau de interesse dos estudantes de Medicina em seguir carreira na Atenção Básica. Assim procedemos por duas razões. Primeira: por não havermos identificado, na literatura nacional, estudos confiáveis sobre a temática em questão. Segunda: por crermos que estudos acerca da escolha da carreira em MFC realizados em países onde ela já está consolidada podem contribuir para o entendimento do fenômeno do Brasil, possibilitando a estruturação de pesquisas mais específicas para testar, em cenários brasileiros, as principais hipóteses levantadas em estudos estrangeiros.

\section{MATERIAL E MÉTODOS}

Para Nóbrega-Therrien e Therrien ${ }^{36}$ (2004, p. 7), “a finalidade do «estado da questão» é de levar o pesquisador a registrar, a partir de um rigoroso levantamento bibliográfico, como se encontra o tema ou o objeto de sua investigação no estado atual da ciência ao seu alcance". Nesse desiderato, realizamos uma revisão bibliográfica recente (de 2006 e 2005), a fim de encontrar estudos que apontassem variáveis relacionadas com 
a escolha da MFC, ou seu equivalente internacional, pelos estudantes de Medicina. Uma vez que nosso interesse não era fazer um levantamento do estado da arte, mas indicar diretrizes futuras de pesquisa, limitamo-nos aos dois anos anteriores à concepção do artigo.

Foi realizada uma busca na Biblioteca Virtual em Saúde (BVS), utilizando como descritores de assunto: "medicina de família" ou "medicina de família e comunidade" e "escolha da profissão" ou "área de atuação profissional" ou "exercício profissional" ou "habilitação profissional" ou "prática profissional" ou "qualificação profissional" ou "educação profissional em saúde pública" ou "profissões" ou "profissões em saúde". Foram encontradas 37 referências publicadas em 2005 e 2006, nos idiomas "inglês", "português" ou "espanhol", das quais 27 correspondiam aos objetivos deste trabalho. Não foram utilizados descritores em inglês, pois a BVS os inclui automaticamente, por meio dos Descritores em Ciências da Saúde (DeCS). Foram excluídos artigos que, apesar de apresentarem as palavras-chave mencionadas, não abordavam o interesse do estudante de Medicina em seguir carreira na MFC ou no equivalente internacional desta especialidade.

Infelizmente, não encontramos, a partir do procedimento empreendido, artigos nacionais voltados para esta temática. Este achado é corroborado por buscas feitas por um dos autores quando da revisão de literatura de sua dissertação de mestrado, utilizando um período de dez anos. Mesmo as referências internacionais não foram, em sua maioria, originadas de pesquisas empíricas. Isto restringiu a análise, na maioria das vezes, a coletar hipóteses levantadas pelos autores, mesmo que não houvesse comprovação científica. Apesar dessas limitações, comentaremos algumas das principais hipóteses para a redução da procura das residências em MFC.

\section{RESULTADOS E DISCUSSÃO}

Como já referido, foram incluídos 27 artigos (Tabela 2). Apenas 33\% das referências coletadas são artigos originados de pesquisas empíricas. As demais são ensaios, cartas ou editoriais (Figura 1). Quanto ao país de origem, 18 referências $(66,7 \%)$ são dos Estados Unidos. As demais são do Canadá (3), Grécia (2), Austrália (2) Inglaterra (1) e Portugal (1).

O motivo mais recorrente para a desmotivação em seguir carreira em Medicina de Família é a baixa remuneração. Essa é a realidade dos Estados Unidos, Canadá, Austrália e Portugal $^{31,32,34,37-41}$. No Brasil, não é diferente. O médico de família tem como principal campo de atuação o PSF, que oferece boa remuneração, mas o salário final deste profissional é inferior ao que alcançam outros especialistas. Entretanto, ainda são necessários estudos que mostrem inequivocamente este fato.
TABELA 2

Periódicos dos artigos incluídos no estudo

\begin{tabular}{lcc}
\hline Periódico & $\begin{array}{c}\text { Fator de } \\
\text { Impacto* }\end{array}$ & $\begin{array}{c}\text { Número } \\
\text { de Artigos }\end{array}$ \\
\hline Academic Medicine & 2,607 & 2 \\
Acta Médica Portuguesa & não encontrado & 1 \\
American Family Physician & 1,616 & 1 \\
Annals of Internal Medicine & 14,780 & 2 \\
Australian Journal of Rural Health & não analisado & 1 \\
Canadian Family Physician & 0,701 & 3 \\
Family Medicine & 1,289 & 2 \\
Family Practice & 1,558 & 1 \\
Family Practice Management & não encontrado & 2 \\
Health Policy & 1,201 & 1 \\
Journal of Rural Health & 1,038 & 1 \\
Journal of the National Medical & & 1 \\
$\quad$ Association & não encontrado & 2 \\
Medical Education & 2,467 & 1 \\
Medical Journal of Australia & 2,582 & 2 \\
Minnesota Medicine & não encontrado & 1 \\
Mont Sinai Journal of Medicine & 1,063 & 2 \\
New England Journal of Medicine & 51,296 & 1 \\
Saudi Medical Journal & não encontrado & \\
\hline
\end{tabular}

* Journal Citation Report 2006. Busca realizada no Portal Capes

\section{FIGURA 1}

Distribuição das referências por categoria.

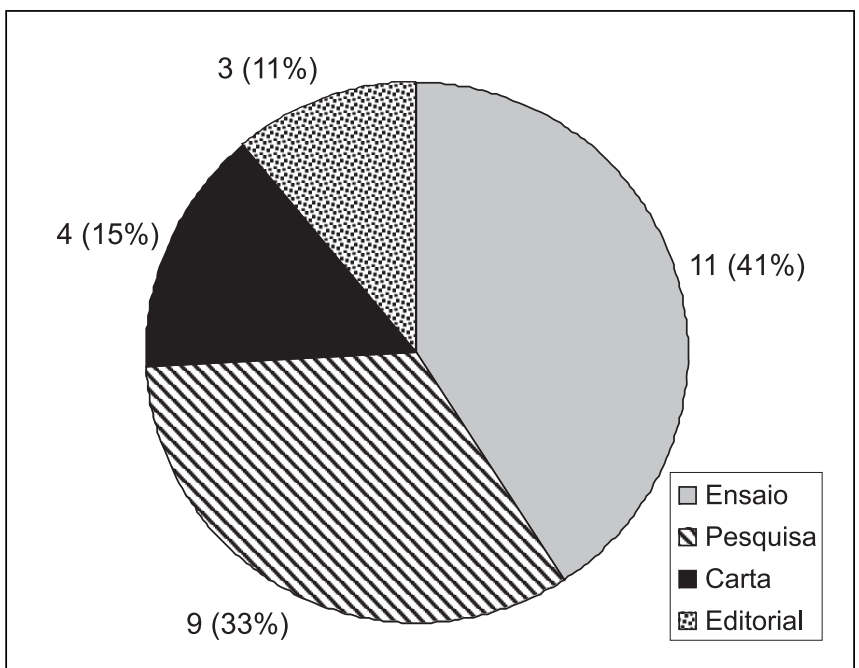

Outro aspecto bastante citado é o pouco prestígio da Medicina de Família. Na Grécia, um estudo realizado em quatro das sete grandes escolas médicas, envolvendo 591 estudantes dos seis anos do curso, mostrou que $49,2 \%$ destes acreditavam que a Medicina de Família não tem prestígio ${ }^{33}$. Em Portugal, um artigo de revisão mostra que a remuneração e o prestígio são variáveis correlacionáveis negativamente com a escolha da Medicina de Família. 
A dívida com a universidade é um motivo de preocupação para os estudantes americanos, o que influencia a escolha por especialidades com maior remuneração ${ }^{31}$. No Brasil, em $2004,51 \%$ dos estabelecimentos de ensino médico eram privados, havendo uma tendência de predomínio dessa categoria administrativa na formação das profissões da área da saúde, principalmente nas regiões Sul e Sudeste ${ }^{42}$. É necessário avaliar, em nossa realidade, a influência dos gastos com a graduação na escolha da carreira.

TABELA 3

Hipóteses para o desinteresse pela especialização em Medicina de Família

Hipótese $^{1}$
Pouco prestígio da Medicina
de Família
de Família

\section{Autores}

Avgerinos, Msaouel, Koussidis et al., 2006 ${ }^{33}$; Hueston, 2006 ${ }^{38}$; Pugno, Schmittling, Fetter Jr. et al., 2005 ${ }^{31}$; Joyce \& Mcneil, 2006 32 ; Gaspar, $2006^{34}$

Pouca vivência em Atenção Primária durante a graduação

Baixos salários

Avgerinos, Msaouel, Koussidis et al. 2006 ${ }^{33}$; Wheat, Higginbotham, Yu et al., $2005^{43}$

Landa, 2006 ${ }^{37}$; Hueston, 2006 ${ }^{38}$; Woo 2006 ${ }^{39}$; Bazargan, Lindstrom, Dakak et al., 2006 ${ }^{40}$; Pugno, Schmittling, Fetter Jr. et al., 2005 ${ }^{31}$; Joyce \& Mcneil, 2006 ${ }^{32}$; Bhyat, 2006 $6^{41}$; Gaspar, $2006^{34}$

Elevada dívida com a universidade

Perfil tecnológico e científico de outras especialidades Amplitude de conhecimento necessária para exercer a especialidade

Perfil socioeconômico elevado Bhyat, $2006^{41}$ dos estudantes de Medicina

Ideia equivocada de que a

Medicina de Família é fácil

demais para o estudante

motivado

Experiência em Medicina de Família com um profissional desmotivado e frustrado

*As hipóteses não foram ordenadas por importância.

Outras variáveis associadas à não escolha da MFC são: (a) perfil tecnológico e científico de outras especialidades ${ }^{32}$, que influencia o prestígio perante a sociedade; (b) perfil socioeconômico elevado dos estudantes de Medicina ${ }^{41}$, que certamente pretendem manter o status quo; (c) pouca vivência em Atenção Primária durante a graduação ${ }^{33,43}$; ou, por outro lado, (d) experiência em Medicina de Família com um profissional desmotivado e frustrado ${ }^{31,34}$; (e) amplitude de conhecimento necessária para exercer a especialidade ${ }^{31,41}$, por um lado e (f) ideia equivocada de que a Medicina de Família é fácil demais para o estudante motivado, por outro ${ }^{31,38}$. Estes achados estão sintetizados na Tabela 3.

\section{CONCLUSÕES: DIRETRIZES FUTURAS PARA PESQUISA}

Quais as diretrizes futuras para pesquisa? Devemos "reconhecer a necessidade de dados objetivos sobre os fatores que influenciam a escolha da especialidade no atual ambiente médico e econômico" ${ }^{\prime 4}$, além de produzir estudos nacionais para avaliar a adequação das hipóteses, por nós relatadas, à realidade brasileira.

A Agenda de Pesquisa a ser construída pode se pautar nas seguintes questões, a partir das hipóteses levantadas anteriormente: (a) até que ponto a concepção dos pais dos acadêmicos acerca dos diferentes níveis de prestígio das especialidades influencia a escolha da carreira?; (b) como os estudantes de Medicina, assim como seus professores, percebem o grau de prestígio da MFC?; (c) em que dimensão há diferenças de remuneração entre as diversas áreas da Medicina?; (d) há influência dos custos com a formação em instituições privadas?; (e) o nível socioeconômico elevado realmente determina a escolha de especialidades com maiores perspectivas salariais?; (f) como os alunos percebem o grau de dificuldade em exercer a MFC e em que medida isso facilita ou dificulta a opção por essa especialidade?; (g) qual a carga horária vivenciada no ambiente de ABS e que repercussões essa experiência teve na forma com que os graduandos encaram a MFC?

Para isto, deve-se lançar mão de métodos quantitativos e qualitativos. Por exemplo, seria interessante avaliar, à semelhança dos americanos, o perfil dos médicos que ingressam nos programas de residência, enfocando desde aspectos socioeconômicos até a categoria administrativa da instituição de formação. De posse desses dados, por meio de análises estatísticas, seria possível reconhecer variáveis que contribuem para a escolha da especialidade a ser seguida. Outra opção seriam questionários, que poderiam empregar escala de Likert, ou, simplesmente, itens predeterminados.

No que concerne aos métodos qualitativos, eles seriam essenciais na abordagem de questões para as quais os métodos quantitativos são insuficientes, nos âmbitos ontológico, epistemológico e metodológico. De fato, os métodos qualitativos têm sido reconhecidos como relevantes para a pesquisa em saúde $^{45}$, bem como para a pesquisa em educação médica ${ }^{46}$. 
Enfim, uma agenda de pesquisa em bases teórico-conceituais e metodológicas adequadas deve ser estabelecida para a problemática levantada neste artigo, a fim de se traçar estratégias para que não "testemunhemos os anos finais da Medicina de Família" como advertiu Dawalt ${ }^{47}$.

\section{REFERÊNCIAS}

1. Anderson MIP, Demarzo M, Rodrigues RD. A Medicina de Família e Comunidade, a Atenção Primária à Saúde e o Ensino de Graduação: recomendações e potencialidades. 2007; [documento on-line].[ocenoem:]. Disponível em http://www.sbmfc.org.br/Articles/Documentos/ 4537dc13/A_medicina_de_familia_\%20-MFC_na_Graduacao_SBMFC.pdf.

2. World Organization of Family Doctors. A Definição Européia de Medicina Geral e Familiar. 2002; http://www. sbmfc.org.br/Articles/Documents/cc20bdb1/Definicao\% 20Europeia\%20de $\% 20$.

3. Atun R. A Experiência da Grã-Bretanha. In: Bessa O, Penaforte J. Médico de Família: formação, certificação e educação continuada. Fortaleza: Escola de Saúde Pública do Ceará; 2002 p.17-43.

4. Ross W. A Experiência do Canadá. In: Bessa O, Penaforte J. Médico de Família: formação, certificação e educação continuada. Fortaleza: Escola de Saúde Pública do Ceará; 2002 p. 45-69.

5. Sloane P. A Experiência dos Estados Unidos. In: Bessa O, Penaforte J. Médico de Família: formação, certificação e educação continuada. Fortaleza: Escola de Saúde Pública do Ceará; 2002 p. 71-98.

6. Falk JW. A medicina de família e comunidade e sua entidade nacional: histórico e perspectivas. Rev. Bras. Med. Fam e Com. 2004; 1: 5-10.

7. Falk JW. Histórico da Especialidade Medicina de Família e Comunidade (MFC) no Brasil. Revista HCPA. 2006; 26(supl 1): 64-65; http://www.hcpa. ufrgs.br/downloads/RevistaCientifica/2006/ANAIS\%202006.pdf

8. Sociedade Brasileira de Medicina de Família e Comunidade. Breve histórico da MFC no Brasil. 2006; http:/ / www. sbmfc.org.br/site/sbmfc/historia.asp

9. Brasil. Ministério da Saúde. Programa Saúde da Família: Como Começou [online]. 2004; http://dtr2001.saude.gov. br/psf//programa/como_comecou.asp

10. Teixeira CF. Saúde da Família, Promoção e Vigilância: construindo a integralidade da atenção à saúde no SUS. Revista Brasileira de Saúde da Família. 2004; 7: 10-23.

11. Brasil. Ministério da Saúde. Política nacional de atenção básica. Brasília: Ministério da Saúde; 2006.
12. Forster AC. Considerações sobre a Formação em Medicina de Família e Atenção Primária. Medicina-Ribeirão Preto. 2001; 34: 202-3.

13. Otero A. La Incorporacion de la Medicina de Família em la Enseñanza de la Medicina em España. Medicina-Ribeirão Preto. 2001; 34: 200-1.

14. Carraccio C, Wolfsthal SD, Englander R, Ferentz K, Martin C. Shifting paradigms: from Flexner to competencies. Acad. Med. 2002; 77:361-67.

15. Marsiglia RG. Relação ensino/serviços: dez anos de integração docente assistencial (IDA) no Brasil. São Paulo: Hucitec; 1995.

16. Feuerwerker LCM. Mudanças na educação médica \& residência médica no Brasil. São Paulo: Hucitec; 1998.

17. Feuerwerker LCM. Além do discurso de mudança na educação médica: processos e resultados. São Paulo: Hucitec; 2002.

18. Almeida M, Feuerwerker L, Llanos M. A educação dos profissionais de saúde na América Latina: teoria e prática de um movimento de mudança, tomo I: um olhar analítico. São Paulo: Hucitec; 1999.

19. Almeida M, Feuerwerker L, Llanos M. A educação dos profissionais de saúde na América Latina: teoria e prática de um movimento de mudança, tomo II: as vozes dos protagonistas. São Paulo: Hucitec; 1999.

20. Almeida MJ. A educação médica e as atuais propostas de mudança: alguns antecedentes históricos. Rev. Bras. Educ. Med. 2001; 25: 42-52.

21. Lampert JB. Tendências de mudanças na formação médica no Brasil: tipologia das escolas. São Paulo: Hucitec; 2002.

22. Ceccim RB, Feuerwerker LCM. Mudança na graduação das profissões de saúde sob o eixo da integralidade. Cadernos de Saúde Pública. 2004; 20: 1400-1410.

23. Brasil. Ministério da Educação e Cultura. CNE/CES/MEC. Resolução no 4, de 7 de novembro de 2001.

24. Brasil. Ministério da Saúde, Ministério da Educação. Portaria Interministerial no 2.101, de 03 de novembro de 2005 . Institui o Programa Nacional de Reorientação da Formação Profissional em Saúde - Pró-Saúde - para os cursos de graduação em Medicina, Enfermagem e Odontologia. Diário Oficial da União, Poder Executivo, Brasília, DF, 4 nov. 2005.

25. Brasil. Ministério da Saúde, Ministério da Educação. Portaria Interministerial no 2.118, de 03 de novembro de 2005 . Institui parceria entre o Ministério da Educação e o Ministério da Saúde para cooperação técnica na formação e desenvolvimento de recursos humanos na área da saúde. 
Diário Oficial da União, Poder Executivo, Brasília, DF, 4 nov. 2005.

26. Magzoub ME, Schmidt HG. A Taxonomy of CommunityBased Medical Education. Acad. Med. 2000; 75: 699-707.

27. Gil CRR. Formação de recursos humanos em saúde da família: paradoxos e perspectivas. Cad. Saúde Pública. 2005; 21: 490-8.

28. Sociedade Brasileira de Medicina de Família e Comunidade. Projeto de Expansão da Residência em Medicina de Família e Comunidade. 2005; http:/ /www.sbmfc.org.br/ site/not/download/Projeto\%20de\%20Expansão\%20dos \%20PRMMFC\%20-\%20SBMFC.pdf.

29. Araújo GG. O que Pensam os Estudantes de Medicina da Universidade Federal do Ceará sobre o Exercício da Profissão Médica. [monografia] Sobral: Escola de Formação em Saúde da Família Visconde de Sabóia; 2001.

30. Cabral Filho WR, Ribeiro VMB. A Escolha Precoce da Especialidade pelo Estudante de Medicina: um Desafio para a Educação Médica. Rev. Bras. Edu. Med. 2004; 28: 133-44.

31. Pugno PA, Schmittling GT, Fetter Jr GT et al. Results of the 2005 National Resident Matching Program: Family Medicine. Family Medicine. 2005; 37: 555-64.

32. Joyce CM, Mcneil JJ. Fewer medical graduates are choosing general practice: a comparison of four cohorts, 1980-1995. Medical Journal of Australia 2006; 185: 102-104.

33. Avgerinos ED, Msaouel P, Koussidis GA et al. Greekmedical students' career choices indicate strong tendency towards specialization and training abroad. Health Policy 2006; 79: 101-106.

34. Gaspar D. Medicina Geral e Familiar: uma escolha gratificante. Acta Méd. Port. 2006; 19: 133-9.

35. Mackean P, Gutkin C. Fewer medical students selecting family medicine: can family practice survive? Canadian Family Physician. 2003; 49: 408-409.

36. Nóbrega-Therrien, S.M.; Therrien, J. Trabalhos científicos e o estado da questão: reflexões teórico-metodológicas. Estudos em Avaliação Educacional, v. 15, n. 30, p. 5-16, JulhoDezembro 2004.

37. Landa AS. Retooling Family Medicine. Minnesota Medicine. 2006; 89: 28-33.

38. Hueston WJ. Rekindling the fire of family medicine. Fam. Pract. Manag. 2006; 13: 15-17.

39. Woo B. Primary Care: the best job in medicine? New England Journal of Medicine. 2006; 355: 864-6.

40. Bazargan M, Lindstrom RW, Dakak A et al. Impact of desire to work in underserved communities on selection of specialty among fourth-year medical students. JAMA 2006; 98: 1460-5.
41. Bhyat R. Where have all the residents gone? Part 1: putting the declining family medicine match rates into context. Can. Fam. Physician 2006; 52: 805-7.

42. Bueno RRL, Pieruccini MC. Abertura de Escolas de Medicina no Brasil: relatório de um cenário sombrio. 2 ed. São Paulo: AMB; Brasília: CFM; 2005.

43. Wheat JR, Higginbotham JC, Yu J et al. Physicians for rural America: the role of institutional commitment within academic medical centers. Journal of Rural Health. 2005; 21: 221-7.

44. Pugno PA, Schmittling GT, Kahn NB. Entry of US Medical School Graduates into Family Medicine Residencies: 2004-2005 and 3-year summary. Family Medicine, 2005; 37: 546-54.

45. Pope C; Mays N. Pesquisa qualitativa na atenção à saúde. Porto Alegre: Artmed; 2005.

46. Stacy R, Spencer J. Assessing the evidence in qualitative medical education research. Medical Education. 2000; 34: 498-500.

47. Dawalt J. Where's the fire? Fam. Pract. Manag. 2006; 13:16.

\section{CONTRIBUIÇÃO DOS AUTORES}

O autor Pedro Gomes Cavalcante Neto executou a revisão da literatura e análise dos dados como parte de sua dissertação de mestrado e, posteriormente, participou da consecução do artigo. Geison Vasconcelos Lira participou na formulação do esqueleto do artigo e da interpretação dos dados, além de escrever partes do artigo. Alcides Silva de Miranda foi orientador da dissertação de mestrado,de Pedro Gomes Cavalcante Neto, tendo orientado todo o processo de revisão de literatura, análise e interpretação dos dados durante a dissertação e durante a confecção do artigo.

\section{CONFLITO DE INTERESSES}

Declarou não haver.

\section{ENDEREÇO PARA CORRESPONDÊNCIA}

Pedro G. Cavalcante Neto Universidade Federal do Ceará, Campus Sobral Curso de Medicina.

Rua Geraldo Rangel, 186

Derby - Sobral

CEP. 62041-040 CE

E-mail: pedro@ufc.br 